\title{
ARTICLE OPEN Atomistic origin of the passivation effect in hydrated silicate glasses
}

\author{
Tao $\mathrm{Du}^{1,2,3}$, Hui Li $\mathbb{D}^{2}$, Qi Zhou ${ }^{3}$, Zhe Wang ${ }^{3}$, Gaurav Sant ${ }^{4,5}$, Joseph V. Ryan ${ }^{6}$ and Mathieu Bauchy ${ }^{3}$
}

When exposed to water, silicate glasses and minerals can form a hydrated gel surface layer concurrent with a decrease in their dissolution kinetics - a phenomenon known as the "passivation effect." However, the atomic-scale origin of such passivation remains debated. Here, based on reactive molecular dynamics simulations, we investigate the hydration of a series of modified borosilicate glasses with varying compositions. We show that, upon the aging of the gel, the passivation effect manifests itself as a drop in hydrogen mobility. Nevertheless, only select glass compositions are found to exhibit some passivation. Based on these results, we demonstrate that the passivation effect cannot be solely explained by the repolymerization of the hydrated gel upon aging. Rather, we establish that the propensity for passivation is intrinsically governed by the reorganization of the medium-range order structure of the gel upon aging and, specifically, the formation of small silicate rings that hinder water mobility.

npj Materials Degradation (2019)3:6 ; https://doi.org/10.1038/s41529-019-0070-9

\section{INTRODUCTION}

When exposed to water, silicate glasses and minerals tend to dissolve via several mechanisms, including hydration, hydrolysis, and ion-exchange. ${ }^{1-3}$ In most cases, the leaching of the mobile cations initially present in the silicate phase (e.g., B and alkali), their replacement by hydrated species, and the restructuring of the leached material results in the formation of a disordered, porous, and hydrated "gel" layer on the surface of the dissolving phase. ${ }^{3,4}$ The formation of this alteration layer is usually associated with a drop in the corrosion rate-a behavior known as the passivation effect. ${ }^{4,5}$ This slowdown in the corrosion kinetics of silicate phases upon their passivation has important consequences in earth science and technological applications involving outdoor silicate phases. ${ }^{6,7}$ In particular, such passivation is expected to largely control the long-term durability of glasses used as a matrix to immobilize nuclear waste. ${ }^{8,9}$

Despite the importance of silicate corrosion, the origin of the passivation effect has thus far remained debated. ${ }^{3}$ The passivation effect is typically understood as (i) a consequence of solution saturation (i.e., a decrease in chemical potential difference between solid and solution), ${ }^{10}$ (ii) the precipitation of amorphous silica following the congruent dissolution of the glass, ${ }^{11-16}$ or (iii) the reorganization of the hydrated gel layer upon aging., ${ }^{2,17-19}$ In the latter case, the passivation effect has been suggested to arise from a decrease in water mobility in the gel or pristine glass. ${ }^{4,5,20,21}$ Nevertheless, the atomic-scale mechanism that governs the passivation effect remains largely unknown.

Although the reorganization of the gel likely cannot explain alone all the features of the passivation effect, ${ }^{20}$ we investigate herein this hypothesis by means of reactive molecular dynamics simulations. Starting from a series of sodium borosilicate glasses with varying compositions, several hydrated gels are prepared by mimicking the leaching of $\mathrm{B}$ and $\mathrm{Na}$ mobile cations and their replacement by hydrated species. We show that the passivation effect manifests itself as a drop in hydrogen mobility upon the aging of the gel. We find that Na-rich glasses feature such passivation effect, whereas B-rich glasses do not. In turn, all hydrated gels are found to exhibit some degree of repolymerization upon aging - which highlights that the passivation effect cannot be solely explained by the repolymerization of the gel. Rather, we demonstrate here that the passivation effect is controlled by the reorganization of the medium-range order structure of the gel upon aging and, specifically, the formation of small silicate rings.

\section{RESULTS}

Parent glasses and preparation of the hydrated gel

To investigate the effect of the composition of the parent glass on the propensity of the resulting hydrated gel to exhibit some passivation, we first simulate using molecular dynamics a series of modified borosilicate glasses $\left(\mathrm{Na}_{2} \mathrm{O}\right)_{0.3-x}(\mathrm{CaO})_{0.1}\left(\mathrm{~B}_{2} \mathrm{O}_{3}\right)_{x}\left(\mathrm{SiO}_{2}\right)_{0.6}$ where $x=0.00,0.05,0.10,0.15,0.20$, and 0.30 (see Methods section). These compositions are intended to offer a simplified model for complex multi-component nuclear waste glasses and to study the effect of the Na-to-B ratio (i.e., the two types of mobile/ soluble cations considered in this series of glasses). ${ }^{8}$ Fig. 1a shows a snapshot of the atomic configuration of a simulated glass comprising $10 \% \mathrm{~B}_{2} \mathrm{O}_{3}$. As expected, we observe a coexistence of three- and four-fold coordinated boron atoms (noted $B^{[3]}$ and $B^{[4]}$ hereafter, respectively), wherein $B^{[4]}$ units are charge-balanced by $\mathrm{Na}$ or $\mathrm{Ca}$ cations (see the inset of Fig. 1a). ${ }^{22}$ In agreement with

\footnotetext{
${ }^{1}$ Key Lab of Structures Dynamic Behavior and Control (Harbin Institute of Technology), Ministry of Education, 150090 Harbin, China; ${ }^{2}$ School of Civil Engineering, Harbin Institute of Technology, 150090 Harbin, China; ${ }^{3}$ Physics of AmoRphous and Inorganic Solids Laboratory (PARISlab), Department of Civil and Environmental Engineering, University of California, Los Angeles, CA 90095, USA; ${ }^{4}$ Laboratory for the Chemistry of Construction Materials (LC2), Department of Civil and Environmental Engineering, University of California, Los Angeles, CA 90095, USA; ${ }^{5}$ California Nanosystems Institute (CNSI), University of California, Los Angeles, CA 90095, USA and ${ }^{6}$ Energy and Environment Directorate, Pacific Northwest National Laboratory, Richland, WA 99352, USA

Correspondence: Hui Li (lihui@hit.edu.cn) or Mathieu Bauchy (bauchy@ucla.edu)
}

Received: 6 November 2018 Accepted: 17 January 2019

Published online: 07 February 2019 
(a)

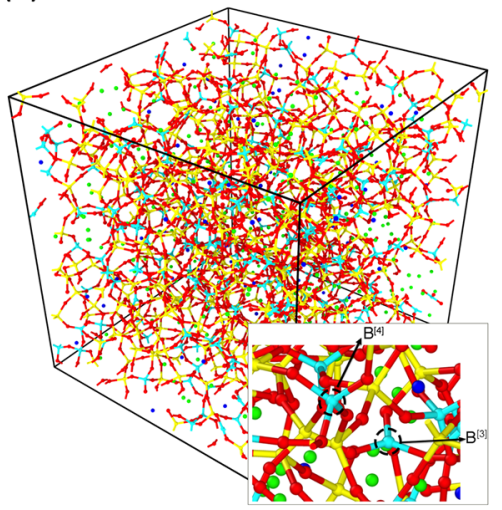

(b)

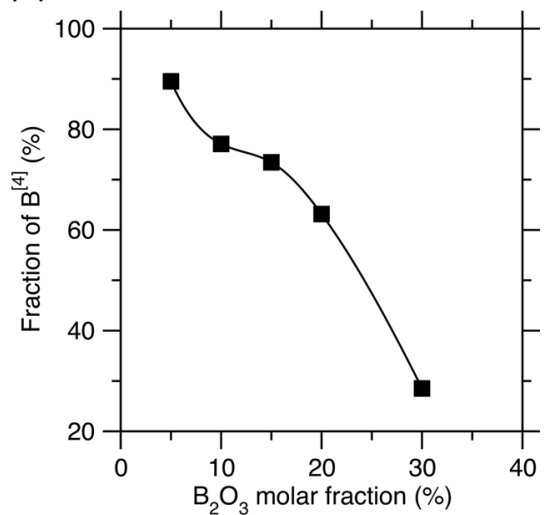

(c)

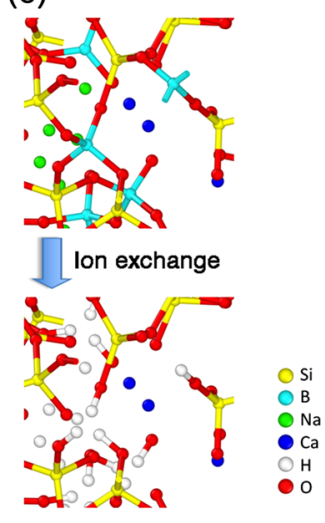

Fig. 1 Structure of the parent glasses and the hydration process. a Representative configuration of an initial parent glass with the following composition: $\left(\mathrm{Na}_{2} \mathrm{O}\right)_{0.2}(\mathrm{CaO})_{0.1}\left(\mathrm{~B}_{2} \mathrm{O}_{3}\right)_{0.1}\left(\mathrm{SiO}_{2}\right)_{0.6}$. The inset highlights the coexistence of three-fold and four-fold boron atoms in the atomic structure of the glass. $\boldsymbol{b}$ Fraction of four-fold coordinated $\mathrm{B}$ atoms in the pristine parent $\left(\mathrm{Na}_{2} \mathrm{O}\right)_{0.3-x}(\mathrm{CaO})_{0.1}\left(\mathrm{~B}_{2} \mathrm{O}_{3}\right)_{x}\left(\mathrm{SiO}_{2}\right)_{0.6} \mathrm{glasses}$ as a function of the molar fraction of $\mathrm{B}_{2} \mathrm{O}_{3}$. c Schematic showing the hydration process (i.e., the leaching of $\mathrm{Na}$ and $\mathrm{B}$, following by their replacement by $\mathrm{H}$ atoms). $\mathrm{Si}, \mathrm{B}, \mathrm{Na}, \mathrm{Ca}, \mathrm{H}$, and $\mathrm{O}$ atoms are here represented in yellow, cyan, green, blue, white, and red, respectively

previous observations, ${ }^{22,23}$ the fraction of $B^{[4]}$ is found to decrease with increasing $x$ (see Fig. 1b), i.e., increasing $\mathrm{B}_{2} \mathrm{O}_{3}$ molar fraction and decreasing $\mathrm{Na}_{2} \mathrm{O}$ molar fraction. This arises from the fact that, as the $\mathrm{B}_{2} \mathrm{O}_{3}$ molar fraction increases, the $(\mathrm{Na}+\mathrm{Ca}) / \mathrm{B}$ ratio decreases, thereby representing a relative decrease in the amount of available charge-compensating ions. ${ }^{22}$ These glasses are then used as "parents" to form some hydrated silicate gels. To this end, we mimic the hydration process by manually replacing the leached species (i.e., $\mathrm{Na}$ and $\mathrm{B}$ ) by $\mathrm{H}$ atoms, while the other elements ( $\mathrm{Si}, \mathrm{Ca}$, and $\mathrm{O}$ ) are retained in the hydrated glass (see Fig. 1c and Methods section). ${ }^{4,24}$ No distinction is made between network-modifying and charge-compensating $\mathrm{Na}$ cations during the leaching phase. Note that Ca cations are not replaced here as this element has been found to be largely retained in the altered gel. ${ }^{24}$ Note that this method does not explicitly simulate the solution or the degree of saturation thereof. Although it would be desirable to explicitly simulate the entire ion exchange process rather than manually replace the leached cations, the timescale of this reaction far exceeds that allowed by MD simulations. As such, our simulations cannot offer any information on the kinetics of the ion exchange process. Nevertheless, the methodology used herein was previously used to simulate ion exchange in silicate glasses (in the context of ion exchange strengthening), wherein the structure and properties of the simulated ion-exchanged glasses were found to be in good agreement with experimental data. ${ }^{25-27}$

\section{Repolymerization of the hydrated gels}

Following the hydration of the glasses, we now study the reorganization of the resulting gels upon accelerated aging (see Methods section). We initially focus on the degree of polymerization of the gel, as captured by the fraction of bridging oxygen atoms (BO, i.e., $\mathrm{O}$ atoms that are connected to two Si or B atomsnote that no $B$ atoms are retained in the gel). First, we note that the degree of polymerization of the hydrated gel decreases with increasing $\mathrm{B}_{2} \mathrm{O}_{3}$ molar fraction in the parent glass (see Fig. 2a,b). This arises from the fact that, initially, (i) the leaching of each $\mathrm{Na}$ cation results in the transformation of a NBO into a silanol group ( $\mathrm{Si}-\mathrm{OH})$ - so that the connectivity remains unchanged, whereas (ii) the leaching of each $\mathrm{B}$ cation involves the transformation of 3 or 4 BOs into silanol groups-which significantly decreases the local connectivity. Second, we observe that, upon aging, the degree of polymerization of the gels tends to increase via recondensation (see Fig. 2a). ${ }^{28}$ Such recondensation occurs via the transformation of two silanol groups into a water molecule. Eventually, the degree of polymerization of the gel appears to plateau after $1 \mathrm{~ns}$ of accelerated aging (see Fig. 2a). Importantly, we observe that all the gels exhibit a comparable extent of repolymerization upon aging (see Fig. 2b), regardless of the composition of the parent glass. Interestingly, after aging, select gels become even more polymerized than their parent glasses-a behavior that is observed here in Na-rich glasses (i.e., $\mathrm{B}_{2} \mathrm{O}_{3}$ molar fraction $<5 \%$ ). Such recondensation of the gel echoes previous experimental observations. ${ }^{5,17,24}$

Further, we observe that the reorganization of the gel upon aging also manifests itself in its overall density. We first note that the density of the parent borosilicate glass increases with increasing $\mathrm{B}_{2} \mathrm{O}_{3}$ molar fraction, in agreement with experimental observations (see Fig. 2c). ${ }^{22,23}$ The leaching of $\mathrm{Na}$ and $\mathrm{B}$ cations then result in a drop in the density of the hydrated gel, as observed experimentally. ${ }^{24}$ This can be explained by the fact that $\mathrm{B}$ and $\mathrm{Na}$ atoms are much heavier than $\mathrm{H}$ atoms. Interestingly, we observe that select gels (i.e., for $\mathrm{B}_{2} \mathrm{O}_{3}$ molar fraction $<20 \%$ ) tend to slightly densify upon aging, whereas others (gels formed from Brich glasses) do not (see Fig. 2c). This highlights the fact that, although all the gels tend to repolymerize over time, the details of the reorganization mechanism appear to be composition-specific.

\section{Water mobility and passivation effect}

We now investigate whether the aged hydrated gels exhibit any signs of a passivation effect. To this end, we study the mobility of hydrogen atoms, which, ultimately, controls the ability of water to diffuse through the gel. ${ }^{29,30}$ Specifically, we characterize the effect of the aging of the gel on water mobility by computing the diffusion coefficient of hydrogen before and after aging (see Methods section and Supplementary Material). ${ }^{31,32}$ Note that, here, thanks to the reactive nature of the present MD simulations, $\mathrm{H}$ atoms can freely dissociate into silanol groups and reform water molecules as they diffuse. To avoid any effect of the timedependent gel reorganization on diffusion, the diffusion simulations are restricted to fairly low temperature, that is, wherein the kinetics of the gel reorganization far exceeds the simulation timescale. Figure $3 a$ shows an example mean-square displacement (MSD) of hydrogen in a hydrated gel (formed from a parent glass comprising $10 \% \mathrm{~B}_{2} \mathrm{O}_{3}$ ), before and after aging. We observe that the MSD exhibit two stages of diffusion, namely, an initial ballistic regime up to about 2 ps, which is followed by the diffusive regime at long time. ${ }^{33}$ These regimes manifest themselves by a slope of 2 and 1 in the log-log plot of the MSD vs. time (see Fig. 3a). ${ }^{31,34}$ Importantly, we observe that the aging of the hydrated gel results in a decrease in the MSD of hydrogen (see Fig. 3a). This 
(a)

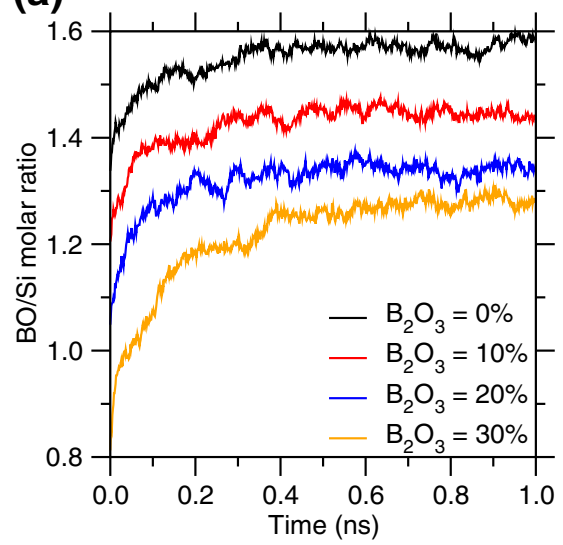

(b)

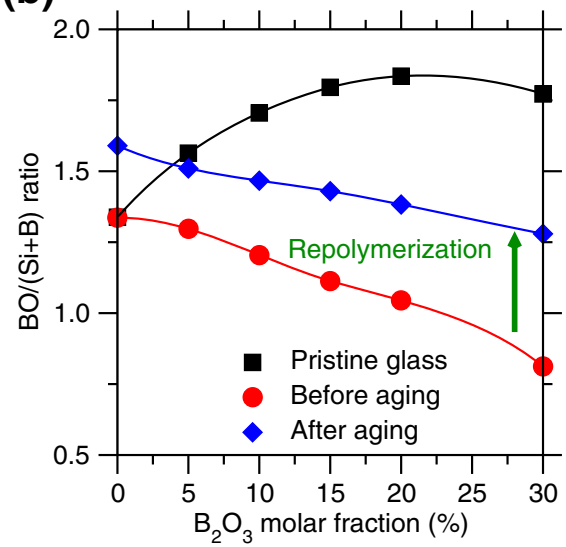

(c)

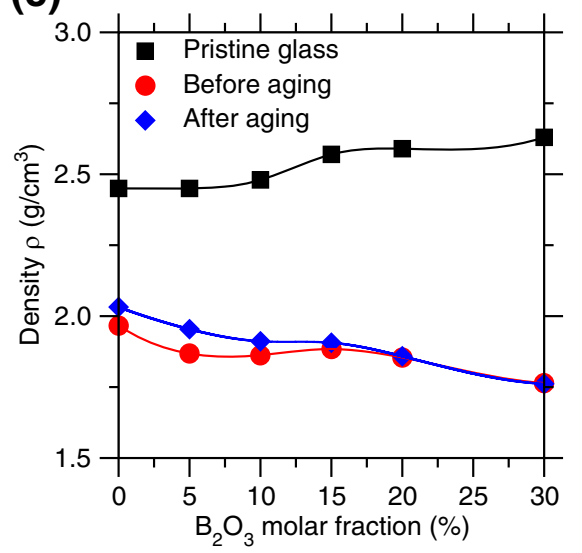

Fig. 2 Structure and density of the parent glasses and the hydrated gels (before and after aging). a Time-dependence of the number of bridging oxygen per $\mathrm{Si}$ atom (BO/Si) during the accelerated aging of the hydrated gels. $\mathbf{b} \mathrm{BO} /(\mathrm{Si}+\mathrm{B})$ molar ratio in the parent glasses and in the hydrated gels (before and after aging) as a function of the $\mathrm{B}_{2} \mathrm{O}_{3}$ molar fraction in the parent glass. c Density of the parent glasses and hydrated gels (before and after aging) as a function of the $\mathrm{B}_{2} \mathrm{O}_{3}$ molar fraction in the parent glass

strongly supports the idea that the passivation effect manifests itself by a decrease in hydrogen mobility in the hydrated gel upon its aging.

To further describe the role of the composition of the parent glass in controlling the propensity of the resulting gel to exhibit a passivation effect, we compute the diffusion coefficient of hydrogen atoms in the hydrated gels at $300 \mathrm{~K}\left(D_{\mathrm{H}}\right)$ and the associated activation energy $\left(E_{\mathrm{A}}\right)$ by relying on an Arrhenius description of the temperature-dependence of diffusion (see Methods section). Figure $3 \mathrm{~b}$ shows the diffusion coefficient of hydrogen in the hydrated gels, before and after aging. We observe that, before aging, the mobility of $\mathrm{H}$ atoms only weakly depends on the composition of the parent glass. In turn, the aging of the gel significantly affects hydrogen mobility. After aging, the diffusion coefficient of hydrogen strongly depends on the composition of the parent glass. Specifically, $D_{\mathrm{H}}$ is found to increase exponentially with increasing $\mathrm{B}_{2} \mathrm{O}_{3}$ molar fraction in the parent glass (see Fig. $3 \mathrm{~b}$ ). Importantly, we observe that the aging of the gel results in a drop in $D_{\mathrm{H}}$ in gels resulting from Na-rich glasses (by up to almost two orders of magnitude), whereas $D_{\mathrm{H}}$ increases upon aging in gels resulting from B-rich glasses. Similarly, as shown in Fig. 3c, we observe that aging results in an increase in the activation energy of diffusion $E_{\mathrm{A}}$ in gels created from Na-rich glasses, whereas $E_{\mathrm{A}}$ decreases upon aging in gels resulting from $B$-rich glasses. Here, an increase in $E_{\mathrm{A}}$ indicates that, on average, hydrogen atoms need to overcome larger energy barriers to jump from one pocket to another. ${ }^{34}$ Altogether, these results indicate that only the gels resulting from $\mathrm{Na}$-rich glasses feature a passivation effect (i.e., a decrease in hydrogen mobility upon aging), whereas those resulting from B-rich glasses do not.

These results are unexpected as dissolution rate and diffusion activation energy have previously been suggested to be controlled by the connectivity of the atomic network. ${ }^{35}$ As such, the fact only select glasses exhibit a decrease in $\mathrm{H}$ mobility contrasts with the fact that all gels tend to repolymerize upon aging (see Fig. 2b). This suggests that the passivation effect cannot be solely explained by the recondensation of the gel upon aging.

Atomistic origin of the passivation effect

The lack of correlation between the degree of repolymerization of a gel upon aging (see Fig. 2b) and its propensity to exhibit a passivation effect (see Fig. 3b) suggests that the drop in hydrogen mobility is not controlled by the short-range connectivity of the gel. Rather, we now focus on the evolution of the medium-range order of the hydrated gels upon aging. In disordered silicate phases, the medium-range order is mostly determined by the ring size distribution-wherein (boro)silicate rings are defined as the shortest closed-path within the $\mathrm{Si}-\mathrm{O} / \mathrm{B}-\mathrm{O}$ network. ${ }^{36}$ Fig. 4a shows an example of ring size distribution in a hydrated gel (derived from the parent glass with $10 \% \mathrm{~B}_{2} \mathrm{O}_{3}$ ), before and after aging (see Methods section and Supplementary Material). Overall, we observe that the ring size distributions exhibit a shape that is similar to those observed in silicate glasses-with an average ring size of 6-7 (see Fig. 4b), wherein the ring size is here defined in terms of the number of $\mathrm{Si}$ and $\mathrm{B}$ atoms belonging to the ring). ${ }^{23,37,38}$ As shown in Fig. $4 \mathrm{~b}$, we observe that the averaging ring size is maximum in the hydrated gels derived from parent glasses comprising around $15 \% \mathrm{~B}_{2} \mathrm{O}_{3}$. This maximum arises from a balance between the two effects of $B$ atoms, namely, they tend to increase the degree of polymerization in the parent glass (by consuming some network-modifying $\mathrm{Na}$ cations and forming $\mathrm{BO}_{4}$ units), whereas, in contrast, their leaching eventually results in a decrease in the degree of polymerization of the gel (with respect to that of the parent pristine glass). Importantly, we note that, upon the aging of the gel, the average ring size tends to decrease in gels resulting from Na-rich glasses (i.e., those that exhibit a passivation effect), whereas it increases in those resulting from Brich glasses (i.e., those that do not exhibit a passivation effect). Overall, the comparison of Figs. $3 \mathrm{~b}, \mathrm{c}$ and $4 \mathrm{~b}$ strongly suggests that the propensity for a hydrated gel to exhibit a passivation effect is controlled by the decrease in its average ring size upon aging. In details, we observe that the ring size distribution exhibits some coarsening (see Fig. 4a), wherein the fractions of small (i.e., 3membered and 4-membered rings) and some of the large rings (6membered and larger rings) increase at the expense of intermediate rings (i.e., the fraction of 5-membered rings decreases). Specifically, the decrease in the average ring size is mostly driven by the formation of a significant fraction of small silicate rings (i.e., 3-membered and 4-membered rings). We also note that, as expected, a decrease in the average ring size results in an increase in density (see Fig. 2c), which is in line with previous results. ${ }^{23}$

Overall, our results suggest that the formation of small silicate rings in the hydrated gels upon aging is the structural origin of the passivation effect. This can be understood from that the fact that such small rings exhibit a lower diameter than their larger counterparts. As such, since hydrated species need to travel through these rings to jump from one pocket to another in the gel, a decrease in ring diameter tends to hinder water mobility. To 
(a)

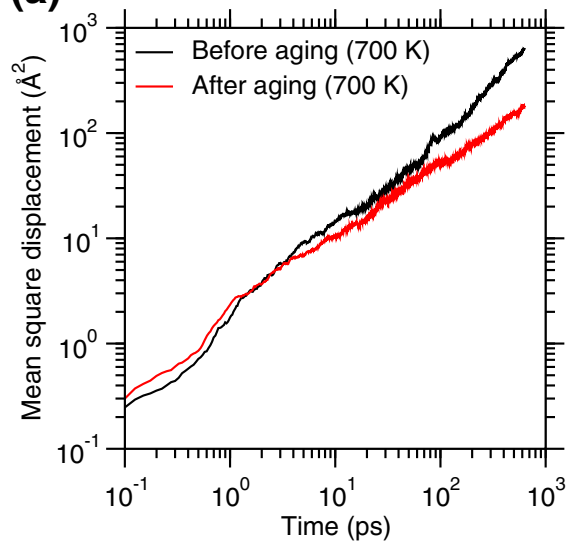

(b)

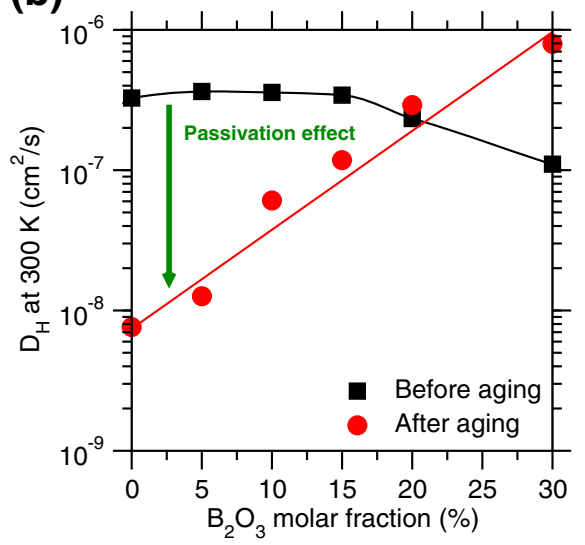

(c)

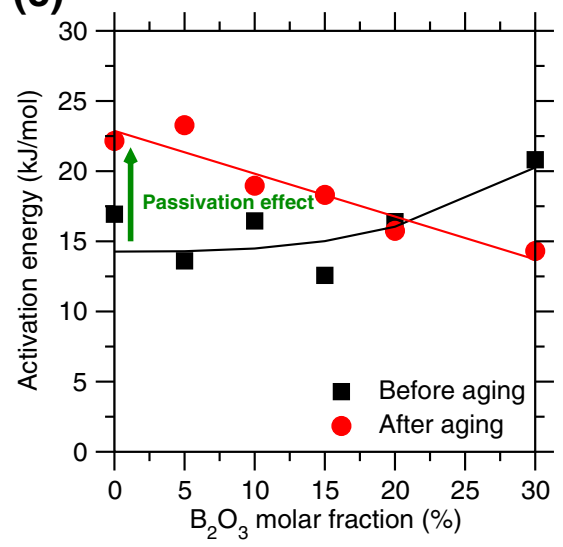

Fig. 3 Water dynamics in the hydrated gels. a Time-dependence of the mean square displacement (MSD) of water molecules in a hydrated gel (derived from a parent glass comprising $10 \% \mathrm{~B}_{2} \mathrm{O}_{3}$ ), before and after aging. $\mathbf{b}$ Diffusion coefficient of the water molecules at $300 \mathrm{~K}$ in the hydrated gels as a function of the initial $\mathrm{B}_{2} \mathrm{O}_{3}$ molar fraction in the parent glasses, before and after the aging of the gels. c Activation energy of water diffusion as a function of the initial $\mathrm{B}_{2} \mathrm{O}_{3}$ molar fraction in the parent glass

confirm this, we compute the structure factors of the hydrated gels before and after aging-i.e., another signature of their medium-range order (see Methods section). ${ }^{39}$ Specifically, the position of the first sharp diffraction peak (FSDP) of the Si-Si partial structure factor has been shown to be inversely correlated with the diameter of the silicate rings. ${ }^{40}$ As shown in Fig. $4 c$, we note that the FSDP of the Si-Si partial structure factor is located around $1.8 \AA^{-1}$, which corresponds to a typical repetition distance of $3.5 \AA$ in real space-i.e., the typical diameter of a fivemembered silicate ring (see Methods section). Importantly, we observe that, upon the aging of the gel, the position of the FSDP increases in gels resulting from $\mathrm{Na}$-rich glasses (i.e., those that exhibit a passivation effect). This confirms that the average diameter of the silicate rings decreases upon the aging of the hydrated gel-which is key in controlling the propensity for passivation of the gel formed from the hydration of alkali-rich (or B-poor) parent glasses.

\section{DISCUSSION}

Finally, we further discuss the relationship between parent glass composition, ring size distribution evolution upon the aging of the gel, and passivation effect. First, we note that the parent glasses virtually do not exhibit any small (boro)silicate rings. ${ }^{23}$ Note that, although the addition of $B$ atoms in the pristine glass tends to increase its initial connectivity (i.e., by consuming some NBOs, see Fig. 2b), this does not result in the formation of small rings. ${ }^{23}$ Starting from this observation, Fig. 5 summarizes the distinct effects of $\mathrm{Na}$ and $\mathrm{B}$ leaching on the ring size distribution in the hydrated gel and, eventually, on the propensity for passivation.

Starting from a B-rich parent glass, the leaching of B cations significantly depolymerizes the glass network and results in the formation of large rings. This arises from the fact that the leaching of $\mathrm{B}$ cations converts some $\mathrm{BO}$ (i.e., $\mathrm{O}$ atoms connected to two $\mathrm{Si}$ or $\mathrm{B}$ atoms) into NBOs (i.e., Si-O-H groups). As the gel ages, some recondensation can occur via the transformation of two silanol groups into a water molecule. However, as shown in Fig. 5a, such recondensation will at best restore some of the rings that preexisted in the parent glass. As such, no small silicate rings silicate rings will be formed.

In contrast, starting from a Na-rich parent glass, the leaching of $\mathrm{Na}$ cations initially does not change the degree of polymerization of the silicate network-since this process simply converts $\mathrm{Si}-\mathrm{O}-\mathrm{Na}$ into $\mathrm{Si}-\mathrm{O}-\mathrm{H}$ bonds. Further, as the gel ages, the mutual recondensation of silanol groups will result in the formation of new rings (see Fig. 5b), that is, rings that did not initially exist in the parent glass. These new rings will necessarily be small (i.e., smaller than the rings initially present in the parent glass) as they form through the subdivision of existing rings.

In turn, when traveling through such small rings, hydrated species need to impose an elastic strain on the ring to accommodate their size, ${ }^{35,41}$ which results in an increase in the activation energy of diffusion (see Fig. $3 \mathrm{c}$ ) and block some of the diffusion pathways for $\mathrm{H}$ atoms. Overall, our results suggest that the loss of water mobility resulting from the formation of small silicate rings upon alkali leaching explains the passivation effect in hydrated silicate glasses. Note that, in addition of the structural evolution of the hydrated gel, the reorganization of the silicate rings in precipitated amorphous silica (if any) may also contribute to the passivation effect. ${ }^{42}$

\section{METHODS}

Preparation of the parent borosilicate glasses

A series of parent borosilicate glasses $\left(\mathrm{Na}_{2} \mathrm{O}\right)_{0.3-x}(\mathrm{CaO})_{0.1}\left(\mathrm{~B}_{2} \mathrm{O}_{3}\right)_{x}\left(\mathrm{SiO}_{2}\right)_{0.6}$ with $x=0 \%, 5 \%, 10 \%, 15 \%, 20 \%$, and $30 \%$ comprising around 3000 atoms are simulated by classical molecular dynamics (see Supplementary Material for more details about the simulated glasses). To this end, we adopt the Wang-Bauchy (WB) potential, which has been shown to offer an excellent description of the structure and properties of borosilicate glasses. ${ }^{23}$ In particular, the WB forcefield allows us to properly predict the average coordination of $B$ atoms over a large range of compositions. ${ }^{23}$ The WB potential relies on a two-body Buckingham formulation with fixed parameters and partial charges:

$U_{i j}\left(r_{i j}\right)=\frac{q_{i} q_{j}}{r_{i j}}+A_{i j} \exp \left(-\frac{r_{i j}}{\rho_{i j}}\right)-\frac{C_{i j}}{r_{i j}^{6}}$

where $r_{i j}$ is the distance between a pair of atoms $i$ and $j, q_{i}$ is the partial charge of atom $i$, and $A_{i j}, \rho_{i j}$, and $C_{i j}$ are the energy parameters for the pair of atoms $(i, j)$. Long-range Coulombic interactions are computed with the PPPM algorithm with an accuracy of $10^{-5} .^{43}$ The cutoff for the short-range and Coulombic interactions are set at $11 \AA \AA$. More details about the parameterization of the WB potential can be found in ref. ${ }^{23}$ and in Supplementary Material.

All the simulations presented herein are carried out using the Largescale Atomic/Molecular Massively Parallel Simulator (LAMMPS) code. ${ }^{44}$ The motion of atoms is described using the velocity-Verlet integration algorithm with a time step of $1 \mathrm{fs}$. The parent borosilicate glass models are prepared with the melt-quenching procedure, ${ }^{45}$ as described in the following. First, an initial configuration is built by randomly placing the atoms into a cubic box while ensuring any unrealistic overlap by using the PACKMOL package. ${ }^{46}$ Then, the system is melted at $4000 \mathrm{~K}$ in the canonical $(N V T)$ ensemble for $200 \mathrm{ps}$ and at zero pressure in the isothermal-isobaric (NPT) ensemble for $100 \mathrm{ps}$-to ensure the loss of the memory of the initial 
(a)

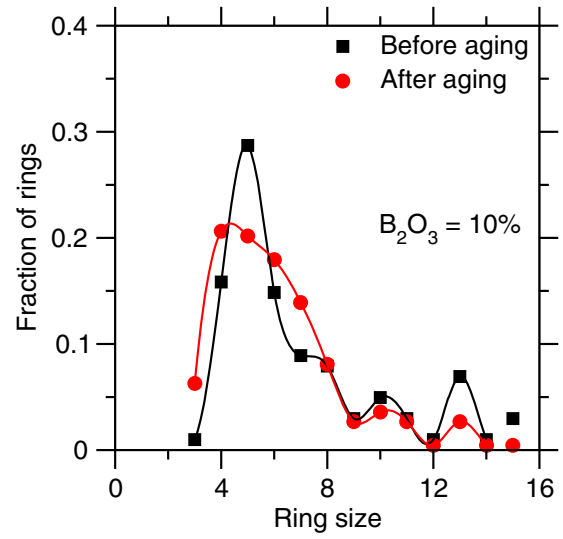

(b)

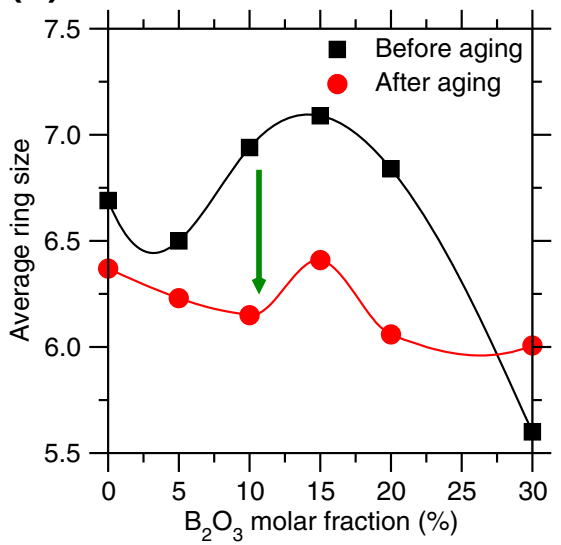

(c)

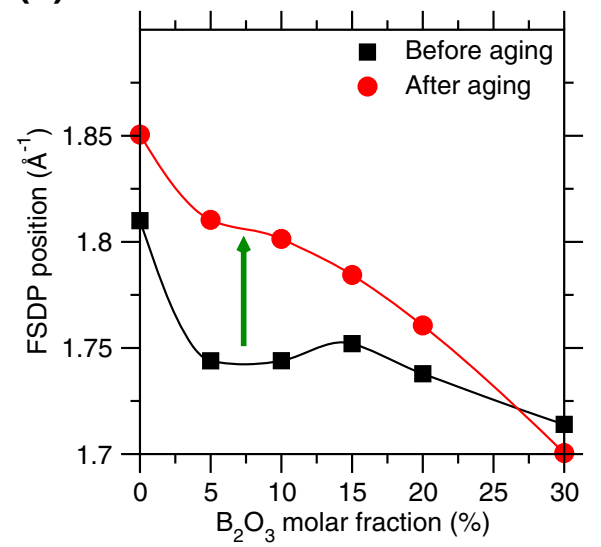

Fig. 4 Medium-range order structure of the hydrated gels. a Example ring size distribution in a hydrated gel (derived from a parent glass comprising $10 \% \mathrm{~B}_{2} \mathrm{O}_{3}$ ), before and after aging-wherein the ring size is defined in terms of the number of Si/B atoms belonging to the ring. $\mathbf{b}$ Average ring size in the hydrated gel as a function of the initial $\mathrm{B}_{2} \mathrm{O}_{3}$ molar fraction in the parent glass, before and after the aging of the gel. $\mathbf{c}$ Position of the first sharp diffraction peak (FSDP) of the Si-Si partial structure factor before and after the aging of the gel as a function of the initial $\mathrm{B}_{2} \mathrm{O}_{3}$ molar fraction in the parent glass

configuration. Subsequently, the system is linearly cooled down to $300 \mathrm{~K}$ at zero pressure with a cooling rate of $1 \mathrm{~K} / \mathrm{ps}$ in the NPT ensemble. Note that, in select cases (e.g., $\mathrm{B}_{2} \mathrm{O}_{3}$ molar fraction $<10 \%$ ), the initial configuration tends to "explode" at high temperature in the NPT ensemble. In such case, the system is initially cooled from 4000 to $2000 \mathrm{~K}$ in the NVT ensemble, equilibrated at $2000 \mathrm{~K}$ in the NPT ensemble under zero pressure, and finally cooled down to $300 \mathrm{~K}$ in the NPT ensemble with a cooling rate of $1 \mathrm{~K} / \mathrm{ps}$. Select structural features of the simulated glasses (partial pair distribution functions (PDF) and bond angle distributions) are presented in Supplementary Material.

\section{Preparation of the hydrated gels}

To describe the hydration of the parent borosilicate glasses, we adopt the reactive forcefield ReaxFF ${ }^{47}$ parametrized by Manzano et al. ${ }^{48}$ ReaxFF is a bond-order-based potential that allows for both the breakage and formation of chemical bond through the calculation of interatomic bond orders-a feature that is required to properly describe the formation hydration of the silicate network and subsequent recondensation. ${ }^{49}$ Additionally, in contrast to conventional classical force fields with fixed atomic charges like the WB potential, ReaxFF dynamically assigns the charges of the atoms based on the charge equilibration method (QEq) which is key to model chemical reactions. ${ }^{50}$ Based on these features, ReaxFF has been shown to properly describe the reactivity of silicate phases while remaining far more computationally efficient than ab initio methods. ${ }^{29,49,51}$ The energy formulation used by ReaxFF consists in several energy terms as follows:

$E_{\text {total }}=E_{\mathrm{bond}}+E_{\mathrm{vow}}+E_{\text {coul }}+E_{\mathrm{under}}+E_{\mathrm{over}}+E_{\mathrm{lp}}+E_{\mathrm{val}}+E_{\mathrm{tors}}+E_{\mathrm{conj}}+E_{\mathrm{pen}}$

where these terms describe, following the order above, the bond energy, Van der Waals energy, Coulombic potential energy, under-coordination energy, over-coordination energy, lone electron pairs energy, valence angle energy, torsion energy, conjugation energy, and penalty energy. The detailed description of these terms can be found in ref. ${ }^{47}$. The ReaxFF parametrization used in this study can be found in Supplementary Material.

The hydrated gels are prepared by subjecting the parent borosilicate glasses to leaching, wherein mobile species ( $\mathrm{B}$ and $\mathrm{Na}$ ) are replaced by $\mathrm{H}$ atoms, whereas the other atoms $(\mathrm{Si}, \mathrm{Ca}$, and $\mathrm{O})$ are retained in place. Specifically, each $\mathrm{Na}$ is manually replaced by $1 \mathrm{H}$, whereas $\mathrm{B}$ is replaced by $3 \mathrm{H}$ atoms - to retain charge neutrality. No undissociated water molecules are initially inserted as the gel initially remains densely packed. Following leaching, the obtained hydrated gel is subjected to an energy minimization and equilibrated at $300 \mathrm{~K}$ for $100 \mathrm{ps}$ in the $N V T$ ensemble and, finally, at zero pressure for $250 \mathrm{ps}$ in the NPT ensemble (using the ReaxFF potential). All the reactive molecular dynamics simulations are carried out using the USER-REAXC package in LAMMPS. ${ }^{52}$

\section{Accelerated aging of the hydrated gels}

The recondensation of silanol groups into water molecules upon the aging of the hydrated gels involves the breaking $\mathrm{Si}-\mathrm{O}-\mathrm{H}$ and formation of $\mathrm{Si}-\mathrm{O}-\mathrm{Si}$ bonds, a process that is associated to high-energy barriers. ${ }^{28}$ As such, the limited timescale of molecular dynamics simulations (i.e., a few $\mathrm{ns}$ ) would not allow for such energy barriers to be overcome. To this end, we here accelerate the aging of the gels by increasing the temperature-a strategy that is commonly used to enhance the sampling kinetics in atomistic simulations. ${ }^{28,53}$ We here adopt a temperature of $2000 \mathrm{~K}$, which was previously found to be high enough to accelerate the polymerization kinetics of silicate gels while remaining low enough not to affect the nature of the condensation mechanism. ${ }^{28}$ The leached hydrated gels are then subjected to accelerated aging by being relaxed at $2000 \mathrm{~K}$ in the NVT ensemble for $1 \mathrm{~ns}$ - which is found to be long enough to achieve a plateau in the degree of polymerization (see Fig. 2a). Following aging, the gels are cooled down to $300 \mathrm{~K}$ with a cooling rate of $10 \mathrm{~K} / \mathrm{ps}$ in the $N V T$ ensemble and subsequently relaxed under zero pressure in the NPT ensemble for 100 ps before any further analysis.

\section{Water mobility}

The mobility of the $\mathrm{H}$ atoms in the hydrated gels-before and after aging -is studied by computing the mean square displacement (MSD) of each $\mathrm{H}$ atom as:

$\operatorname{MSD}_{i}(t)=\Delta r_{i}^{2}(t)=\left|r_{i}(t+\tau)-r_{i}(\tau)\right|^{2}$

where $r_{i}(t)$ is the position of the $i$ th atom at time $t$ and the brackets represent the averaging over multiple time origins $(\tau)$. The self-diffusion coefficient $D_{\mathrm{s}}$ is related to the slope of the MSD vs. time in the diffusion regime and can be computed from the Einstein relationship in 3D:

$$
D_{\mathrm{s}}=\frac{1}{6} \lim _{t \rightarrow \infty} \frac{\operatorname{MSD}(t)}{t}
$$

Note that, to calculate the diffusion coefficient, we ensure that a diffusion regime is achieved (i.e., which manifests itself as a slope of 1 in the log-log plot of MSD vs. time). All MSD calculations are here conducted in the NVT ensemble to avoid any spurious effect due to volume changes. Starting from hydrated gels configurations obtained before and after aging, respectively, the MSD is computed at 500,600, and $700 \mathrm{~K}$ during $600 \mathrm{ps}$ of dynamics. These temperatures are chosen so as be high enough so that the diffusion regime can be achieved, but low enough to prevent any repolymerization of the gel during the timescale of the MSD simulations. The temperature dependence of the computed diffusion 
(a) Leaching of B cations
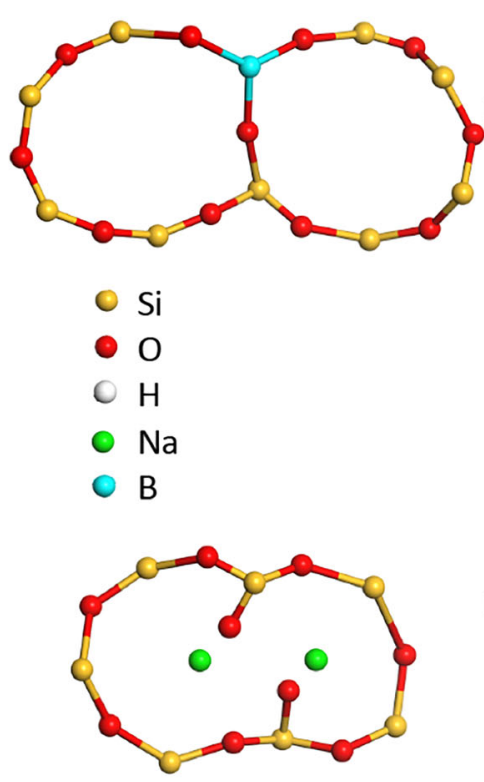

(b) Leaching of Na cations

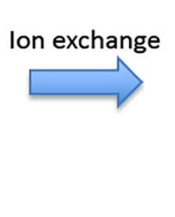

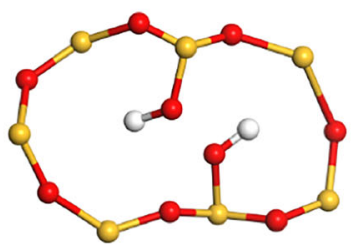

No small rings

No passivation effect

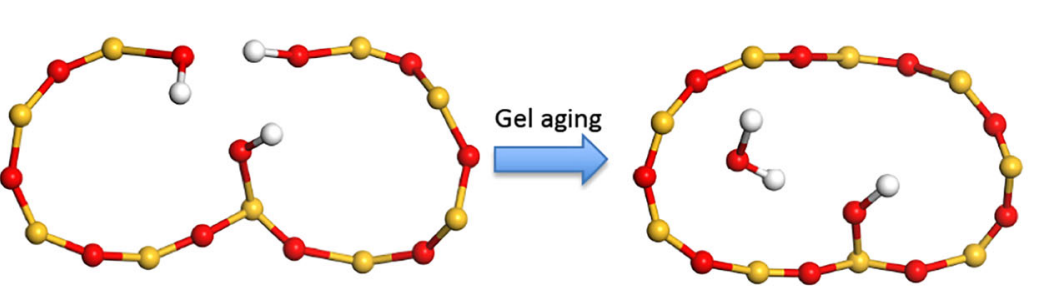

Fig. 5 Schematic showing the effect of ion exchange and subsequent recondensation on the ring size distribution. a Upon the leaching of Na cations. b Upon the leaching of B cations. Si, O, H, Na, and B atoms are represented in yellow, red, white, green, and cyan, respectively. Select surrounding atoms are here omitted for clarity

coefficients is then fitted by an Arrhenius fit:

$D_{\mathrm{s}}=D_{0} \exp \left(\frac{-E_{\mathrm{A}}}{R T}\right)$

to estimate the activation energy of diffusion $\left(E_{\mathrm{A}}\right)$ and, by extrapolation, the diffusion coefficient of the water molecules in the hydrated gels at $300 \mathrm{~K}$.

\section{Coordination analysis}

The $\mathrm{O}$ atoms are distinguished into $\mathrm{BO}$ (i.e., connected to two network formers $\mathrm{Si}$ or $\mathrm{B}$ ) and NBO (i.e., connected to only one network former) based on their local topology. To this end, we first compute the partial PDF for each system and chose the position of the minimum after the first peak of the PDF as the cutoff distinguishing the first from the second coordination shell. The topology of each $\mathrm{O}$ atom is then determined by enumerating the number of $\mathrm{Si}$ and $\mathrm{B}$ atoms present in its first coordination shell. The overall degree of polymerization of the phase is here defined by the number of $\mathrm{BO}$ atoms per network-forming species ( $\mathrm{Si}$ and $\mathrm{B}$ ).

Ring size distribution

The ring size distribution of each system is computed by using the RINGS package ${ }^{54}$ wherein a (boro)silicate ring is defined as the shortest closed paths within the borosilicate skeleton network-that is, excluding network-modifying species and terminating $\mathrm{O}$ atoms. ${ }^{55}$ The ring size is here defined in terms of the number of Si and B atoms they comprise. A maximum ring size of 15 is here adopted, which is found to be high enough for the ring size distribution to converge.

Structure factor and first-sharp diffraction peak

We compute the Faber-Ziman Si-Si partial structure factors $S^{\mathrm{FZ}}{ }_{\mathrm{Sisi}}(Q)$ through the Fourier transform of the Si-Si partial PDF $g_{\mathrm{Sisi}}(r):^{.56}$

$S_{\mathrm{SiSi}}^{\mathrm{FZ}}(Q)=1+4 \pi \rho \int_{0}^{\infty} \mathrm{d} r r^{2} \frac{\sin Q r}{Q r}\left(g_{\mathrm{Sisi}}(r)-1\right)$

where $Q$ is the reciprocal vector and $\rho$ is the atomic density. The first-sharp diffraction peak (FSDP) of the Si-Si partial structure factor is then by fitted by a Lorentzian function-since it has been shown that Lorentzian functions better fit the FSDP of the structure factor of glassy silica than
Gaussian functions. ${ }^{57}$ This fit allows us to extract the position ( $\left.Q_{F S D P}\right)$ of the FSDP - which is found to be in the range of $1.7-1.85 \AA^{-1}$, in agreement with previous experiment and simulation results. ${ }^{58,59}$ The $\mathrm{Si}-\mathrm{Si}$ typical repetition distance in real space is then computed as $d=2 \pi / Q_{\text {FSDP. }}{ }^{60}$

\section{DATA AVAILABILITY}

All data that support the findings of this study are available from the corresponding author upon reasonable request.

\section{ACKNOWLEDGEMENTS}

This research was performed using funding received from the DOE Office of Nuclear Energy's Nuclear Energy University Programs. The authors also acknowledge some financial support provided by the National Science Foundation (Grant No. 1562066) and China Scholarship Council (Grant No. 201706120252).

\section{AUTHOR CONTRIBUTIONS}

M.B. designed the research, T.D. performed the atomistic simulations, T.D., Q.Z., and Z. W. analyzed the simulation results, T.D., H.L, G.S., J.V.R., and M.B. wrote the manuscript.

\section{ADDITIONAL INFORMATION}

Supplementary information accompanies the paper on the npj Materials Degradation website (https://doi.org/10.1038/s41529-019-0070-9).

Competing interests: The authors declare no competing interests.

Publisher's note: Springer Nature remains neutral with regard to jurisdictional claims in published maps and institutional affiliations.

\section{REFERENCES}

1. Varshneya, A. K. Fundamentals of Inorganic Glasses (Academic Press Inc., San Diego, CA, 1993).

2. Bunker, B. C. Molecular mechanisms for corrosion of silica and silicate glasses. J. Non-Cryst. Solids 179, 300-308 (1994). 
3. Vienna, J. D., Ryan, J. V., Gin, S. \& Inagaki, Y. Current understanding and remaining challenges in modeling long-term degradation of borosilicate nuclear waste glasses. Int. J. Appl. Glass Sci. 4, 283-294 (2013).

4. Gin, S. et al. Origin and consequences of silicate glass passivation by surface layers. Nat. Commun. 6, 6360 (2015).

5. Gin, S. et al. Dynamics of self-reorganization explains passivation of silicate glasses. Nat. Commun. 9, 2169 (2018).

6. Scholze, H. Chemical durability of glasses. J. Non-Cryst. Solids 52, 91-103 (1982).

7. Conradt, R. Chemical durability of oxide glasses in aqueous solutions: a review. J. Am. Ceram. Soc. 91, 728-735 (2008).

8. Gin, S. et al. An international initiative on long-term behavior of high-level nuclear waste glass. Mater. Today 16, 243-248 (2013).

9. Jantzen, C. M., Brown, K. G. \& Pickett, J. B. Durable glass for thousands of years. Int. J. Appl. Glass Sci. 1, 38-62 (2010).

10. Grambow, B. Nuclear waste glasses-how durable? Elements 2, 357-364 (2006)

11. Putnis, A. Sharpened interface: glass corrosion. Nat. Mater. 14, 261-262 (2015).

12. Lenting, $C$. et al. Towards a unifying mechanistic model for silicate glass corrosion. Npj Mater. Degrad. 2, 28 (2018).

13. Hellmann, R. et al. Nanometre-scale evidence for interfacial dissolution -reprecipitation control of silicate glass corrosion. Nat. Mater. 14, 307-311 (2015).

14. Geisler, T. et al. Aqueous corrosion of borosilicate glass under acidic conditions: a new corrosion mechanism. J. Non-Cryst. Solids 356, 1458-1465 (2010).

15. Geisler, T. et al. The mechanism of borosilicate glass corrosion revisited. Geochim Cosmochim. Acta 158, 112-129 (2015).

16. Hellmann, R. et al. Unifying natural and laboratory chemical weathering with interfacial dissolution-reprecipitation: a study based on the nanometer-scale chemistry of fluid-silicate interfaces. Chem. Geol. 294-295, 203-216 (2012).

17. Gin, S. et al. Nuclear glass durability: new insight into alteration layer properties. J. Phys. Chem. C. 115, 18696-18706 (2011).

18. $\mathrm{Ma}, \mathrm{T}$. et al. A mechanistic model for long-term nuclear waste glass dissolution integrating chemical affinity and interfacial diffusion barrier. J. Nucl. Mater. 486, 70-85 (2017).

19. Grambow, B. \& Müller, R. First-order dissolution rate law and the role of surface layers in glass performance assessment. J. Nucl. Mater. 298, 112-124 (2001).

20. Gin, S. et al. Atom-probe tomography, TEM and ToF-SIMS study of borosilicate glass alteration rim: a multiscale approach to investigating rate-limiting mechanisms. Geochim. Cosmochim. Acta 202, 57-76 (2017).

21. Cailleteau, C. et al. Insight into silicate-glass corrosion mechanisms. Nat. Mater. 7 , 978-983 (2008).

22. Smedskjaer, M. M. et al. Topological principles of borosilicate glass chemistry. J. Phys. Chem. B 115, 12930-12946 (2011).

23. Wang, M. et al. A new transferable interatomic potential for molecular dynamics simulations of borosilicate glasses. J. Non-Cryst. Solids 498, 294-304 (2018).

24. Collin, M. et al. Structure of International Simple Glass and properties of passivating layer formed in circumneutral pH conditions. Npj Mater. Degrad. 2, 4 (2018).

25. Wang, $M$. et al. lon exchange strengthening and thermal expansion of glasses: common origin and critical role of network connectivity. J. Non-Cryst. Solids $\mathbf{4 5 5}$, 70-74 (2017).

26. Tandia, A., Vargheese, K. D., Mauro, J. C. \& Varshneya, A. K. Atomistic understanding of the network dilation anomaly in ion-exchanged glass. J. Non-Cryst Solids 358, 316-320 (2012).

27. Vargheese, K. D., Tandia, A. \& Mauro, J. C. Molecular dynamics simulations of ionexchanged glass. J. Non-Cryst. Solids 403, 107-112 (2014).

28. Du, T., Li, H., Sant, G. \& Bauchy, M. New insights into the sol-gel condensation of silica by reactive molecular dynamics simulations. J. Chem. Phys. 148, 234504 (2018).

29. Fogarty, J. C., Aktulga, H. M., Grama, A. Y., van Duin, A. C. T. \& Pandit, S. A. A reactive molecular dynamics simulation of the silica-water interface. J. Chem. Phys. 132, 174704 (2010)

30. Rimsza, J. M. \& Du, J. Interfacial structure and evolution of the water-silica gel system by reactive force-field-based molecular dynamics simulations. J. Phys. Chem. C 121, 11534-11543 (2017).

31. Qomi, M. J. A., Bauchy, M., Ulm, F.-J. \& Pellenq, R. J.-M. Anomalous compositiondependent dynamics of nanoconfined water in the interlayer of disordered calcium-silicates. J. Chem. Phys. 140, 054515 (2014).

32. Krishnan, N. M. A. et al. Confined water in layered silicates: the origin of anomalous thermal expansion behavior in calcium-silicate-hydrates. ACS Appl. Mater Interfaces 8, 35621-35627 (2016).

33. Kob, W. Computer Simulations of Supercooled Liquids and Glasses. 32 (Springer, Dordrecht, 2005). https://link.springer.com/chapter/10.1007/978-1-4020-32868166.

34. Bauchy, M. \& Micoulaut, M. From pockets to channels: density-controlled diffusion in sodium silicates. Phys. Rev. B 83, 184118 (2011).

35. Pignatelli, I., Kumar, A., Bauchy, M. \& Sant, G. Topological control on silicates' dissolution kinetics. Langmuir 32, 4434-4439 (2016).
36. Micoulaut, M. \& Phillips, J. C. Rings and rigidity transitions in network glasses. Phys. Rev. B 67, 104204 (2003).

37. Sharma, S. K., Mammone, J. F. \& Nicol, M. F. Raman investigation of ring con figurations in vitreous silica. Nature 292, 140-141 (1981).

38. Krishnan, N. M. A., Wang, B., Le Pape, Y., Sant, G. \& Bauchy, M. Irradiation- vs. vitrification-induced disordering: The case of alpha-quartz and glassy silica. $J$. Chem. Phys. 146, 204502 (2017).

39. Elliott, S. R. Medium-range structural order in covalent amorphous solids. Nature 354, 445-452 (1991)

40. Wang, B. et al. Irradiation-induced topological transition in $\mathrm{SiO}_{2}$ : structural signature of networks' rigidity. J. Non-Cryst. Solids 463, 25-30 (2017).

41. Anderson, O. L. \& Stuart, D. A. Calculation of activation energy of ionic conductivity in silica glasses by classical methods. J. Am. Ceram. Soc. 37, 573-580 (1954).

42. Brinker, C. J. \& Scherer, G. W. Sol-gel-glass.1. Gelation and gel structure. J. NonCryst. Solids 70, 301-322 (1985).

43. Hockney, R. W., Eastwood, J. W. \& Eastwood, J. W. Computer Simulation Using Particles (CRC Press, Boca Raton, 1988).

44. Plimpton, S. Fast parallel algorithms for short-range molecular dynamics. J. Comput. Phys. 117, 1-19 (1995).

45. Li, X. et al. Cooling rate effects in sodium silicate glasses: bridging the gap between molecular dynamics simulations and experiments. J. Chem. Phys. 147, 074501 (2017).

46. Martínez, L., Andrade, R., Birgin, E. G. \& Martínez, J. M. PACKMOL: a package for building initial configurations for molecular dynamics simulations. J. Comput. Chem. 30, 2157-2164 (2009).

47. van Duin, A. C. T., Dasgupta, S., Lorant, F. \& Goddard, W. A. ReaxFF: a reactive force field for hydrocarbons. J. Phys. Chem. A 105, 9396-9409 (2001).

48. Manzano, H., Pellenq, R. J. M., Ulm, F.-J., Buehler, M. J. \& van Duin, A. C. T. Hydration of calcium oxide surface predicted by reactive force field molecular dynamics. Langmuir 28, 4187-4197 (2012).

49. Yu, Y., Krishnan, N. M. A., Smedskjaer, M. M., Sant, G. \& Bauchy, M. The hydrophilic-to-hydrophobic transition in glassy silica is driven by the atomic topology of its surface. J. Chem. Phys. 148, 074503 (2018).

50. Rappe, A. K. \& Goddard, W. A. Charge equilibration for molecular dynamics simulations. J. Phys. Chem. 95, 3358-3363 (1991).

51. Abdolhosseini Qomi, M. J. et al. Combinatorial molecular optimization of cement hydrates. Nat. Commun. 5, 4960 (2014).

52. Aktulga, H. M., Fogarty, J. C., Pandit, S. A. \& Grama, A. Y. Parallel reactive molecular dynamics: Numerical methods and algorithmic techniques. Parallel Comput. $\mathbf{3 8}$ 245-259 (2012)

53. Côté, A. S., Cormack, A. N. \& Tilocca, A. Influence of calcium on the initial stages of the sol-gel synthesis of bioactive glasses. J. Phys. Chem. B 120, 11773-11780 (2016).

54. Le Roux, S. \& Jund, P. Ring statistics analysis of topological networks: New approach and application to amorphous $\mathrm{GeS} 2$ and $\mathrm{SiO}_{2}$ systems. Comput. Mater. Sci. 49, 70-83 (2010).

55. Guttman, L. Ring structure of the crystalline and amorphous forms of silicon dioxide. J. Non-Cryst. Solids 116, 145-147 (1990).

56. Faber, T. E. \& Ziman, J. M. A theory of the electrical properties of liquid metals. Philos. Mag.: A J. Theor. Exp. Appl. Phys. 11, 153-173 (1965).

57. Wright, A. C. et al. The structure of some simple amorphous network solids revisited. J. Non-Cryst. Solids 129, 213-232 (1991).

58. Gaskell, P. H. \& Wallis, D. J. Medium-range order in silica, the canonical network glass. Phys. Rev. Lett. 76, 66-69 (1996).

59. Du, J. \& Corrales, L. R. Compositional dependence of the first sharp diffraction peaks in alkali silicate glasses: a molecular dynamics study. J. Non-Cryst. Solids 352, 3255-3269 (2006).

60. Göttlicher, J. \& Pentinghaus, H. J. Compositional influence on shape and position of the first sharp diffraction peak (FSDP) in silicate and germanate glasses. Ber. Bunsenges. Phys. Chem. 100, 1563-1568 (1996).

\begin{abstract}
Open Access This article is licensed under a Creative Common Attribution 4.0 International License, which permits use, sharing, adaptation, distribution and reproduction in any medium or format, as long as you give appropriate credit to the original author(s) and the source, provide a link to the Creative Commons license, and indicate if changes were made. The images or other third party material in this article are included in the article's Creative Commons license, unless indicated otherwise in a credit line to the material. If material is not included in the article's Creative Commons license and your intended use is not permitted by statutory regulation or exceeds the permitted use, you will need to obtain permission directly from the copyright holder. To view a copy of this license, visit http://creativecommons. org/licenses/by/4.0/.
\end{abstract}

(c) The Author(s) 2019 